\title{
A High Voltage Capacitor Element Model
}

\author{
Calum J. Mackinnon \\ Department of Electronic and Electrical Engineering \\ University of Strathclyde \\ Glasgow, Scotland \\ Calum.Mackinnon@Strath.ac.uk
}

\author{
Brian G. Stewart \\ Department of Electronic and Electrical Engineering \\ University of Strathclyde \\ Glasgow, Scotland \\ Brian.Stewart.100@Strath.ac.uk
}

\begin{abstract}
High voltage capacitors are becoming ever more prevalent on modern electrical power networks, as they offer simple means of power factor correction and voltage support, and are inherent to modern power electronic converter designs. Large capacitor banks comprise many modules, each of which contains an array of individual elements, across which voltage stresses and thermal conditions are shared. A module's partial degradation due to short-circuited elements can increase stresses on the insulation of those that remain, sometimes leading to cascading element failure. This paper presents a high voltage capacitor model, and then explores the distribution of voltage under healthy and short-circuit scenarios. It shows voltage distributions between elements within a capacitor module have nonlinearity due to a module's geometry, and are affected by series element failure.
\end{abstract}

Keywords—Capacitor, Dielectrics, Simulation, High Voltage.

\section{INTRODUCTION}

To accommodate asynchronous generation, an increase in diversity of supply and demand, and to improve resilience, power electronic converters are increasingly being deployed on modern power systems. Such converters are commonplace in: renewable connections, to decouple turbine speed from $\mathrm{AC}$ network frequency; flexible $\mathrm{AC}$ transmission systems such as static compensation (STATCOMs), to improve and control power flows on existing assets; and in high voltage direct current (HVDC) links, to enable controllable, high-capacity connections between assets separated by great distances, sea, and operating frequencies. Availability is desired for these systems to benefit networks and to incentivise investment in further transformative projects. Moreover, since many of these systems are recent, bespoke developments and employ technologies which continue to be refined, reliability information might be limited, commercially sensitive, and in any case is not readily available.

Modern forms of reinforcement such as HVDC and new generation tend to connect by power electronic converters. Since such developments are typically for large capacities, their availability becomes increasingly critical toward maintaining network diversity, providing access to bulk storage, and allowing placement and connection of generation from geographically disparate renewable resources. As this trend toward a greater penetration of renewable generation continues, and potentially as inertia wanes, electrical networks could become all the more reliant on converter-connected systems.

Conventional converter topologies offer high capacities, but introduce characteristic and non-characteristic harmonics to a system. This trend is set to continue and risks exposing existing, potentially aged, assets to greater harmonic distortion. Filtering assets, and those used to support voltage, are therefore central to safeguarding the electrical conditions necessary for converter technologies to contribute to a power network without degrading established (and, depending on where a connection is made, potentially critical) electrical infrastructure. Capacitor banks are one such asset, which play a dual role in HVDC filters as a component of filters and in supporting reactive power, and for which at present there remains a limited academic literature [1].

Rather than await statistical information on asset reliability, it is preferable for the engineering community to anticipate degradation and failure of certain assets. One approach to understanding how assets might degrade over time is by employing representative models, which can simulate stresses to emulate the process of breakdown. Simulated asset models can additionally be used to investigate the influence of assets' operational environment and their dynamic characteristics, which could deviate from measurements acquired offline under static circumstances. While modern converter topologies are increasingly sought, conventional options remain commercially sensible alternatives for high-capacity connections to strong networks. It is therefore possible that any refinement in asset designs could be considered in systems yet to be commissioned.

Section II provides context for the model introduced in Section III, including some of its features. Section IV will then present the results, which are discussed in Section V. Section VI proffers conclusions and indicates future work.

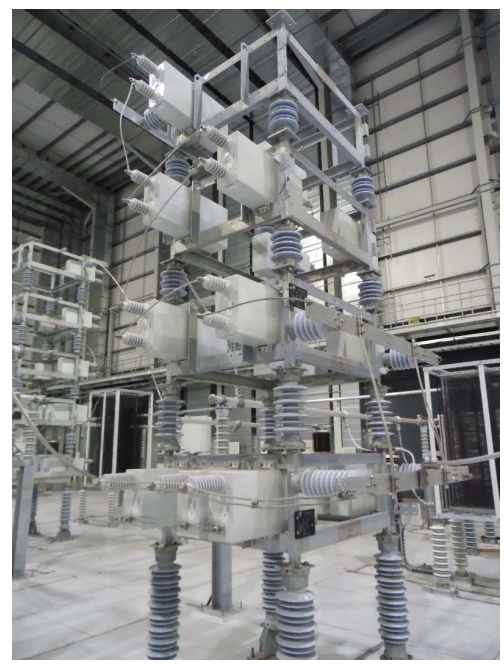

Fig. 1. A capacitor bank at an HVDC converter station. 


\section{BACKGROUND}

Dielectric integrity defines longevity of many electrical assets and plays a "major role" as a primary component in high voltage capacitors [2]. Dielectric strength can be characterised by relative permittivity, a function of voltage amplitude, frequency, and temperature [3].

As seen in Fig. 1, a capacitor bank consists of a number of individual modules connected in series or parallel: the amount of capacitance connected in parallel permits support for reactive power, whereas the capacitance connected in series determines the maximum voltage a capacitor bank is able to withstand [2]. Racks of a capacitor bank are insulated from one another on account of the fact that each is at a different electrical potential with respect to adjacent racks, to reduce the voltage step from the asset structure and the electrical signal to which it connects.

Module design and construction is described in [2] and [4]. Modules are typically formed by layering strips of aluminium foil between a polypropylene dielectric. These layers are rolled to form a single capacitive element, leaving an area of foil exposed at either end, and are then stacked and compressed to fit within a module housing. Adjacent rolls are connected by the exposed foil terminals on each, firstly in parallel to form 'series sections', and these series sections are in turn are connected in series throughout a module. As applies to complete capacitor banks, capacitances in parallel support reactive power, and those in series accommodate voltage. Aluminium foils can be $12 \mu \mathrm{m}$ thick, multiple layers of dielectric material can be combined to a thickness of $20 \mu \mathrm{m}$ [5], and some commercially available modules have outer housing only $1.5 \mathrm{~mm}$ thick. Capacitor modules' outermost dimensions vary to accommodate electrical, thermal dissipation, and space requirements on site. Dielectrics also vary, and while polypropylene film is common, modern dielectric fluids are often proprietary, and selected for fire and environmental safety.

Key design aspects are reliability and longevity, and a selection of fusing arrangements are possible. Modules can be: externally or internally fused, fuseless, or 'self-healing' in which metallized electrodes are vaporised by the arc of a dielectric breakdown, thereby isolating the failed film and permitting most of a series element to remain active. 'Self-healing' designs are robust, but unsuitable for high-power applications due to overheating and thermal gradients [5]. Instead, high-power applications more commonly employ internally fused designs [6], [7]. Where fuses isolate part of a module, a terminal voltage is then shared among remaining elements, and in turn, increased voltage stresses can provoke further breakdown and encourage cascading effects [4].

\section{A CAPACITOR MODEL}

The capacitor model illustrated in Fig. 2 is developed as a basis for simulations in Comsol Multiphysics. This geometry is intended to be simple to permit any problems in its development to be easily uncovered and reduce simulation times, while being amenable to accommodating element numbers representative of real assets and allowing general principles of operation to be explored. It contains only ten aluminium foils, arranged as a simplified 'Swiss-roll' design, visible within a cross-section of the geometry presented in Fig. 3.

\section{A. Mesh}

To reduce simulation execution times, an efficient mesh has been specified cognisant of two particulars of the geometry: narrowly spaced, thin foils, and a thin outer housing. While a simplified geometry is relatively impervious to longer execution times imposed by less efficient meshing, it becomes important to use an efficient mesh when a model scales to be representative of real assets with their associated detail. The mesh for this capacitor model is consequently designed in four layers, where each forms the central volume of the subsequent layer:

1) Foils Layer - an innermost section applies a mapped mesh to foil surfaces, which is swept to fill volumes between them to create a central array of anisotropic elements, sized according to user specified parameters;

2) Growth Layer - to expand a mesh from narrowly-spaced elements on foils, to more isotropic and reasonably sized elements, a mapped mesh is defined with a scaling factor appropriate to the mesh defined on foils, and is swept across each outward face of this layer;

3) Outer Housing - an outer housing is also thin at $1.5 \mathrm{~mm}$, and as such is conducive to a mapped and swept mesh to maintain overall mesh efficiency, as is the case on foils;

4) Dielectric Fill - a free tetrahedral mesh joins the swept meshing on the growth layer to that on the outer housing.

Combined, these four mesh 'layers' create an efficient and reasonable quality mesh on the geometry's outer housing and central foils, between which a limited volume of free meshing is cultivated to provide continuity. This approach intends to reduce simulation time, permit increased numbers of foil elements, and adapt to user preferences for mesh fidelity.

\section{B. Materials}

Materials are selected from built-in options where available: steel is used for module housing and peripherals such as rack mounts; foils are aluminium; and a user-defined material is applied as a dielectric between foils and their enclosure of 3.9 relative permittivity. This can be amended via a user parameter.

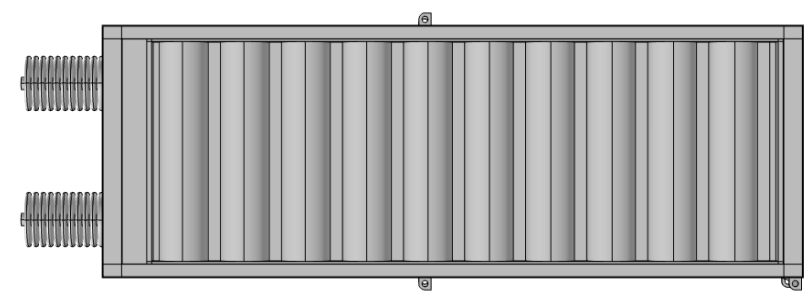

Fig. 2. A simplified geometry

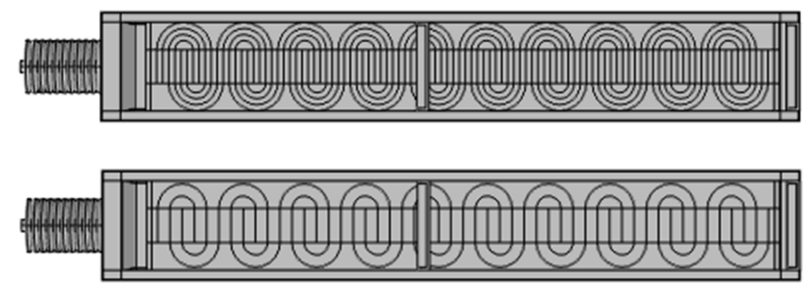

Fig. 3. A cross-section of the model geometry 


\section{Parameterisation}

Parameters allow the model to be adapted to suit different capacitor sizes and foil configurations, and to adapt mesh and dielectric conditions from a common point within the simulation environment. In turn this can facilitate comparisons between module sizes, configurations, and meshing. Furthermore, parameterisation: aids readability, maintainability, and extensibility of the model file itself; can be used to encapsulate the model within Comsol's 'Application Builder' facility such that it can be run outwith the usual simulation environment; and an ability to interface model parameters with a larger system could allow eventual real-world data to inform a hybrid data fusion model to compare with and replicate measured behaviour.

\section{Finite versus Boundary Element Modelling}

Boundary element modelling (BEM) is an alternative to finite element modelling (FEM) and is useful in electrostatics simulations since it allows continuous results to be obtained. A cleaner geometry of two-dimensional foils can be created with BEM, as illustrated by Fig. 3, which can assist the model to scale to accommodate a greater number of capacitive elements than would be possible with a FEM simulation alone. Section IV, however, provides results for the case of modelling with FEM.

\section{Simulation StUdiES AND RESUlts}

Simulation studies have been undertaken based on the model presented in Section III. Two boundary conditions are configured within the simulation environment: one represents electrical connectivity between a signal source and elements; and another simulates the behaviour of the asset model given its specified geometry and materials. A $50 \mathrm{~Hz}, 10 \mathrm{kV}$ supply signal at $1 \Omega$ impedance is applied, which provides initial results: voltages throughout the geometry (and at each foil) are shown in Fig. 4 and Fig. 5, and relative to a linear distribution in Fig. 6. Table I lists voltages at each foil, for 11 scenarios to show how any short-circuit between foils can affect voltage. Terminals 0 and $10(\mathrm{~T} 0, \mathrm{~T} 10)$ consistently hold $0 \mathrm{~V}$ and $10 \mathrm{kV}$, respectively.

TABLE I. SHORT-CIRCUIT FOIL VOLTAGES AT $50 \mathrm{~Hz}$

\begin{tabular}{|l|c|c|c|c|c|c|c|c|c|}
\hline \multirow{2}{*}{$\begin{array}{c}\text { Short } \\
\text { Circuit }\end{array}$} & \multicolumn{7}{|c|}{ Foil Voltages (V) } \\
\cline { 2 - 10 } & T1 & T2 & T3 & T4 & T5 & T6 & T7 & T8 & T9 \\
\hline None & 454.39 & 1176.5 & 2062.3 & 3045.1 & 4089.3 & 5172.6 & 6299.3 & 7477.6 & 8703.8 \\
\hline T0-T1 & 0 & 504.84 & 1317.1 & 2313.8 & 3424.9 & 4607.6 & 5855.4 & 7170.6 & 8544.8 \\
\hline T1-T2 & 451.04 & 451.04 & 1238.6 & 2228.3 & 3343.1 & 4536.0 & 5798.2 & 7130.7 & 8524.0 \\
\hline T2-T3 & 468.74 & 1171.1 & 1171.1 & 2122.7 & 3228.0 & 4428.7 & 5709.7 & 7067.7 & 8490.9 \\
\hline T3-T4 & 496.99 & 1254.9 & 2119.8 & 2119.8 & 3183.8 & 4370.9 & 5654.9 & 7026.1 & 8468.3 \\
\hline T4-T5 & 521.86 & 1329.6 & 2276.6 & 3247.2 & 3247.2 & 4386.3 & 5649.2 & 7015.0 & 8460.6 \\
\hline T5-T6 & 539.91 & 1384.0 & 2391.0 & 3456.4 & 4497.6 & 4497.6 & 5705.8 & 7041.7 & 8471.1 \\
\hline T6-T7 & 553.67 & 1424.9 & 2476.0 & 3610.1 & 4758.6 & 5854.0 & 5854.0 & 7121.3 & 8506.1 \\
\hline T7-T8 & 564.07 & 1454.9 & 2536.2 & 3715.3 & 4931.6 & 6131.2 & 7274.2 & 7274.2 & 8574.7 \\
\hline T8-T9 & 570.69 & 1473.6 & 2573.0 & 3778.1 & 5032.3 & 6289.0 & 7521.1 & 8687.0 & 8687.0 \\
\hline T9-T10 & 573.45 & 1481.5 & 2589.0 & 3805.8 & 5077.8 & 6361.7 & 7637.0 & 8872.6 & 10000 \\
\hline
\end{tabular}

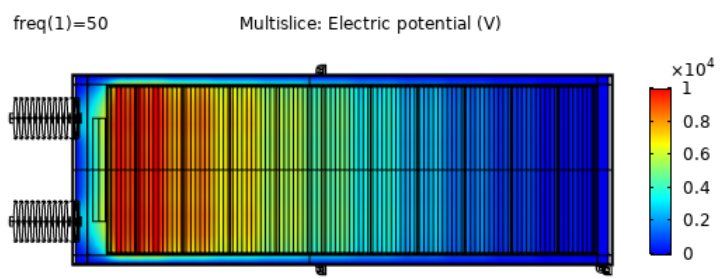

Fig. 4. A distribution of voltage over a simple module cross-section
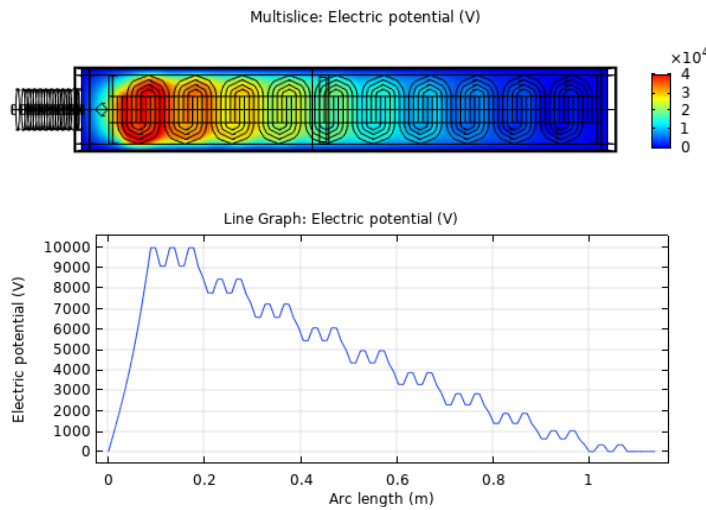

Fig. 5. Voltage over a cross-section and cut line of a simple module

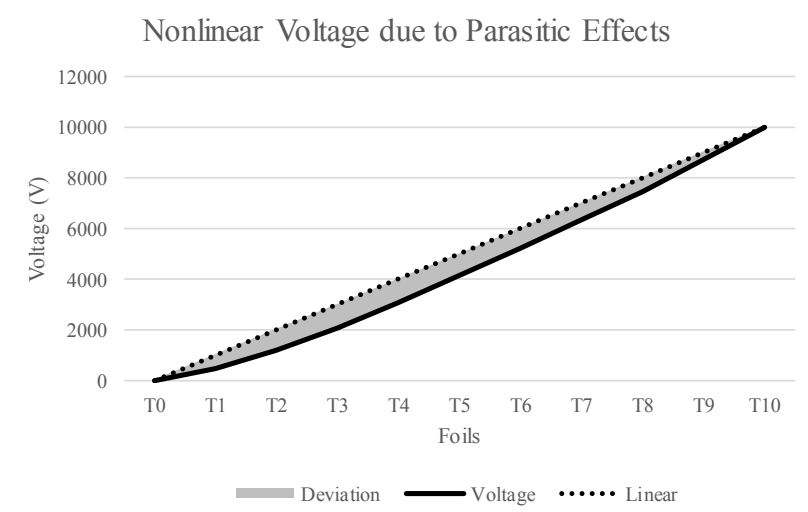

Fig. 6. Nonlinear voltage due to parasitic effects

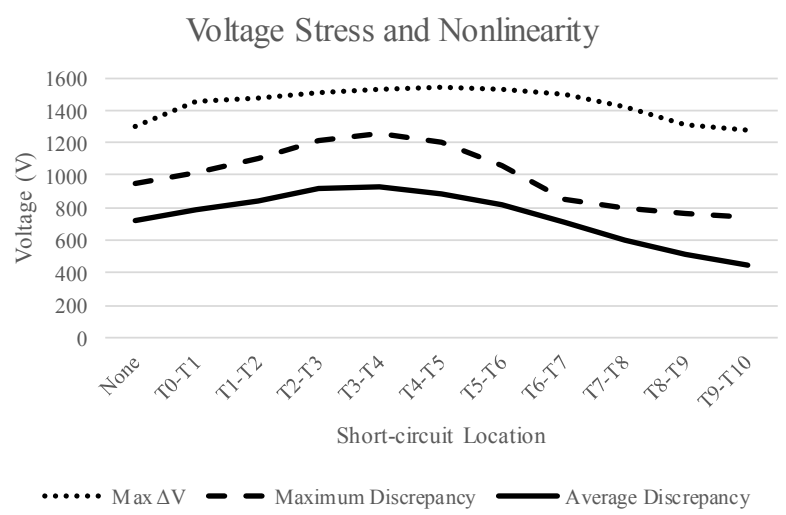

Fig. 7. Voltage stresses between foils and deviation from a straight line. 


\section{DISCUSSION AND IMPLICATIONS}

Each row of Table I corresponds to a single short-circuit scenario, beginning with an absence of any short-circuit and where each pair of adjacent foils is short-circuited in turn. Each column corresponds to voltages at each of ten discrete foils in the model. The results are provided graphically in Fig. 4 and Fig. 5 , which show the distribution of voltage within a capacitor module to be nonlinear, as is more clearly illustrated in Fig. 6.

Since there is variation neither in the geometry between any two foils nor in the dielectric permittivity throughout the model, this nonlinearity must intrinsically result from the capacitor module geometry. While capacitances arise between adjacent foils, as expected, potential differences between each foil and a 'grounded' module housing also invites a parasitic capacitance associated with each foil: one that connects to ground in parallel with all lower-voltage foils and their own stray capacitances; this cumulative effect provokes a subtle nonlinearity.

The extent that a voltage distribution deviates from a linear relationship, for each short-circuit simulation scenario listed in Table I, can be evaluated by the integral between them, indicated by the area highlighted between the curve and line of Fig. 6, or otherwise compared by the average and maximum discrepancy from a linear distribution and the corresponding maximum voltage stress $(\Delta \mathrm{V})$ between any two adjacent foils as indicated in Fig. 7. Such parameters describe a module's ability to evenly distribute voltage and mitigate the chance of further accelerated dielectric aging which can lead to cascading series element failures [4]; any nonlinear distribution suggests that some elements experience greater voltage stresses than others, with consequent predisposition to degradation and eventual failure.

Fig. 7 shows that a short-circuit increases maximum voltage stress between any remaining pair of adjacent foils compared to a healthy asset, save for failure of the highest-voltage element. In this anomalous circumstance the voltage distribution throughout a module is more linear, as is the case for any shortcircuit occurring above the sixth foil. Short-circuits below this foil lead to greater average and maximum discrepancies from a linear voltage distribution.

These results might offer a mild reassurance, given that a healthy asset experiences highest voltage stresses between its highest voltage foils. However, caution is due since this study does not consider the influence of greater total reactance [2], and therefore greater voltage, as could occur in the event of an element failure on a real power system. In this model, an applied voltage remains unaltered between scenarios. The geometry itself is similarly simplistic since it contains only a limited number of foil elements, and omits: elements connected in parallel; effects of temperature; and the effects of a capacitor bank structure, each of which would influence a real system. A change in reactance from series element failure could in turn lead to filter detuning, and compromise capacitor banks' ability to support reactive power and correct power factors.

Furthermore, a dielectric's characteristic eigen frequency and temperature dictate how frequency components in the applied signal influence its relative permittivity [3], which will in turn affect capacitances and voltage distributions throughout a module. Such considerations present an opportunity for more detailed future study, particularly for scenarios which include harmonics characteristic of common converter topologies.

\section{CONCLUSIONS}

Primarily, this paper presents a rudimentary capacitor model with which simulation studies can be run. It is scalable through meshing techniques; adaptable through parameters; and while imperfect, offers a means of simulation toward understanding dynamic asset behaviour under operational conditions.

Preliminary results presented in Section IV suggest that voltages are distributed nonlinearly throughout a capacitor module, possibly resulting from parasitic capacitances which arise as a function of the module geometry. Following a shortcircuit, the voltage distributed across remaining foils is also nonlinear, and this distribution varies as a function of the location of the failed capacitor element. Higher voltage foils experience the greatest voltage stresses, but simulation results suggest that subsequent voltage stress increases (and therefore further propensity for dielectric degradation and breakdown) are overall most exacerbated when lower voltage elements fail.

This model is limited by its simplicity, but it offers a basis on which such considerations can be explored in future work, for which the capacitor model can be scaled to better represent real assets, and for which different geometries, electrical frequencies, and thermal constraints can be considered.

Security, diversity, and cost of an electrical power system depend on the health, reliability, and longevity of its constituent assets. An improved understanding of capacitor behaviour can help inform operation and maintenance decisions in the context of a wider electrical power system. Preliminary results obtained by simulation indicate potential differences between internal foils and an external module housing likely effect nonlinear voltage distributions in a high voltage capacitor element model.

\section{REFERENCES}

[1] H. Jouybari-Moghaddam, T. S. Sidhu, M. R. Dadash Zadeh, P. P. Parikh, "Shunt capacitor banks online monitoring using a superimposed reactance method”, IEEE Trans. Smart Grid, Vol. 9, No. 6, pp. 5554-5563, 2018.

[2] S. Samineni, C. Labuschagne, J. Pope, "Principles of shunt capacitor bank application and protection", $63^{\text {rd }}$ Annual Conference for Protective Relay Engineers, 2010.

[3] R. Arora, W. Mosch, High Voltage and Electrical Insulation Engineering. John Wiley and Sons, Inc.: New Jersey, 2011, pp. 8.

[4] M. Ellis, D. J. Meisner, M. Thakur, "Innovative protection schemes for H configuration fuseless grounded shunt capacitor banks", $65^{\text {th }}$ Annual Conference for Protective Relay Engineers, pp. 449-458, 2012.

[5] J. B. Ennis, F. W. MacDougall, R. A. Cooper, J. Bates, "Repetititve pulse application of self-healing high voltage capacitors", Conference Record of the Twenty-fifth International Power Modulator Symposium and HighVoltage Workshop, pp. 634-638, 2002.

[6] M. A. El-Hadidy, D. H. Helmi, "Failure analysis of medium voltage capacitor banks: the Egyptian experience", $20^{\text {th }}$ International Conference and Exhibition on Electricity Distribution, June 2009.

[7] H. Jouybari-Moghaddam, T. S. Sidhu, M. R. Dadash Zadeh, P. P. Parikh "Enhanced fault-location scheme for double wye shunt capacitor banks", IEEE Trans. Power Delivery, Vol. 32, No. 4, pp. 1872-1880, Aug. 2017. 